\title{
Octreotide use for rescue of vision in a pregnant patient with acromegaly
}

\section{Anne Marie Hannon'1, Isolda Frizelle1, George Kaar², Steven J Hunter ${ }^{3}$, Mark Sherlock4, Christopher J Thompson ${ }^{4}$ and Domhnall J O'Halloran ${ }^{1}$ on behalf of the Irish Pituitary Database Group}

Departments of ${ }^{1}$ Endocrinology and Diabetes, ${ }^{2}$ Neurosurgery, Cork University Hospital, Cork, Ireland, ${ }^{3}$ Department of Endocrinology and Diabetes, Royal Victoria Hospital, Belfast, UK, and ${ }^{4}$ Department of Endocrinology and Diabetes, Beaumont Hospital, Dublin, Ireland
Correspondence should be addressed to A M Hannon Email annemarie_hannon@ hotmail.com

\section{Summary}

Pregnancy in acromegaly is rare and generally safe, but tumour expansion may occur. Managing tumour expansion during pregnancy is complex, due to the potential complications of surgery and side effects of anti-tumoural medication. A 32-year-old woman was diagnosed with acromegaly at 11-week gestation. She had a large macroadenoma invading the suprasellar cistern. She developed bitemporal hemianopia at 20-week gestation. She declined surgery and was commenced on $100 \mu \mathrm{g}$ subcutaneous octreotide tds, with normalisation of her visual fields after 2 weeks of therapy. She had a further deterioration in her visual fields at 24-week gestation, which responded to an increase in subcutaneous octreotide to $150 \mu \mathrm{g}$ tds. Her vision remained stable for the remainder of the pregnancy. She was diagnosed with gestational diabetes at 14/40 and was commenced on basal bolus insulin regimen at 22/40 gestation. She otherwise had no obstetric complications. Foetal growth continued along the 50th centile throughout pregnancy. She underwent an elective caesarean section at 34/40, foetal weight was $3.2 \mathrm{~kg}$ at birth with an APGAR score of 9 . The neonate was examined by an experienced neonatologist and there were no congenital abnormalities identified. She opted not to breastfeed and she is menstruating regularly post-partum. She was commenced on octreotide LAR $40 \mathrm{mg}$ and referred for surgery. At last follow-up, 2 years post-partum, the infant has been developing normally. In conclusion, our case describes a first presentation of acromegaly in pregnancy and rescue of visual field loss with somatostatin analogue therapy.

\section{Learning points:}

- Tumour expansion may occur in acromegaly during pregnancy.

- Treatment options for tumour expansion in pregnancy include both medical and surgical options.

- Somatostatin analogues may be a viable medical alternative to surgery in patients with tumour expansion during pregnancy.

\section{Background}

Acromegaly is a rare disease characterised by excessive growth hormone production. Subfertility is common in acromegaly and has various aetiologies, both as a result of the pituitary adenoma and also due to the treatments involved to treat the disease resulting in hypogonadism.
Pregnancy in acromegaly has been reported to be generally safe $(1,2,3)$; however, rarely tumour expansion may occur. As this is a rare occurrence, little is known regarding the optimum management of these patients during pregnancy. Here, we present the first 
case of resolution of a visual field defect with the use of somatostatin analogues during pregnancy.

\section{Case presentation}

A 32-year-old woman presented at 11-week gestation of a planned pregnancy to her local hospital with headaches. She had a past medical history of asthma and depression. A CT scan of the brain was performed due to claustrophobia and a pituitary lesion was identified. She was referred to her regional pituitary centre and was noted to have enlarged hands, prognathism and increased inter-dentate spacing. She denied any visual disturbance and formal visual field testing confirmed intact fields. She had no family history of pituitary disease.

\section{Investigation}

MRI of her pituitary gland without contrast confirmed a $3.0 \times 2.6 \times 2.6 \mathrm{~cm}$ mass with significant distortion of the infundibulum and optic chiasm (Fig. 1A). Her plasma GH concentration was significantly elevated at $65 \mu \mathrm{g} / \mathrm{L}$ following a glucose load at 12 weeks. Her plasma glucose concentrations were in the normal range, her prolactin was $827 \mathrm{mU} / \mathrm{L}$ (reference value in non-pregnant females:102-496 mU/L - ) and her plasma IGF-1 concentration (IDS-iSYS) was also elevated at $82 \mathrm{nmol} / \mathrm{L}$ (normal range: 9-33 nmol/L). Her plasma GH concentration was measured using the IDS-iSYS assay, which is specific for $22 \mathrm{kDa}$ pituitary GH.

Her morning cortisol was $116 \mathrm{nmol} / \mathrm{L}$ and due to difficulty with interpreting ACTH axis testing during pregnancy, it was deemed safest shecommence replacement dose glucocorticoid treatment for presumptive ACTH deficiency. Biweekly visual field testing was arranged to monitor for tumour expansion. Her baseline blood results are summarised in Table 1.

\section{Treatment}

At 20/40 gestation she was reviewed in the pituitary clinic and complained of increased headaches. Repeat noncontrast MRI showed a slight increase in the size of the adenoma at $3.2 \times 2.7 \times 2.8 \mathrm{~cm}$ with significant compression of the infundibulum and optic chiasm (Fig. 1B). Formal perimetry testing revealed bitemporal hemianopia (Fig. 2). Surgical and medical options were discussed with the patient. The patient declined surgical intervention and opted for medical therapy. Both cabergoline and octreotide therapies were considered, however, in view
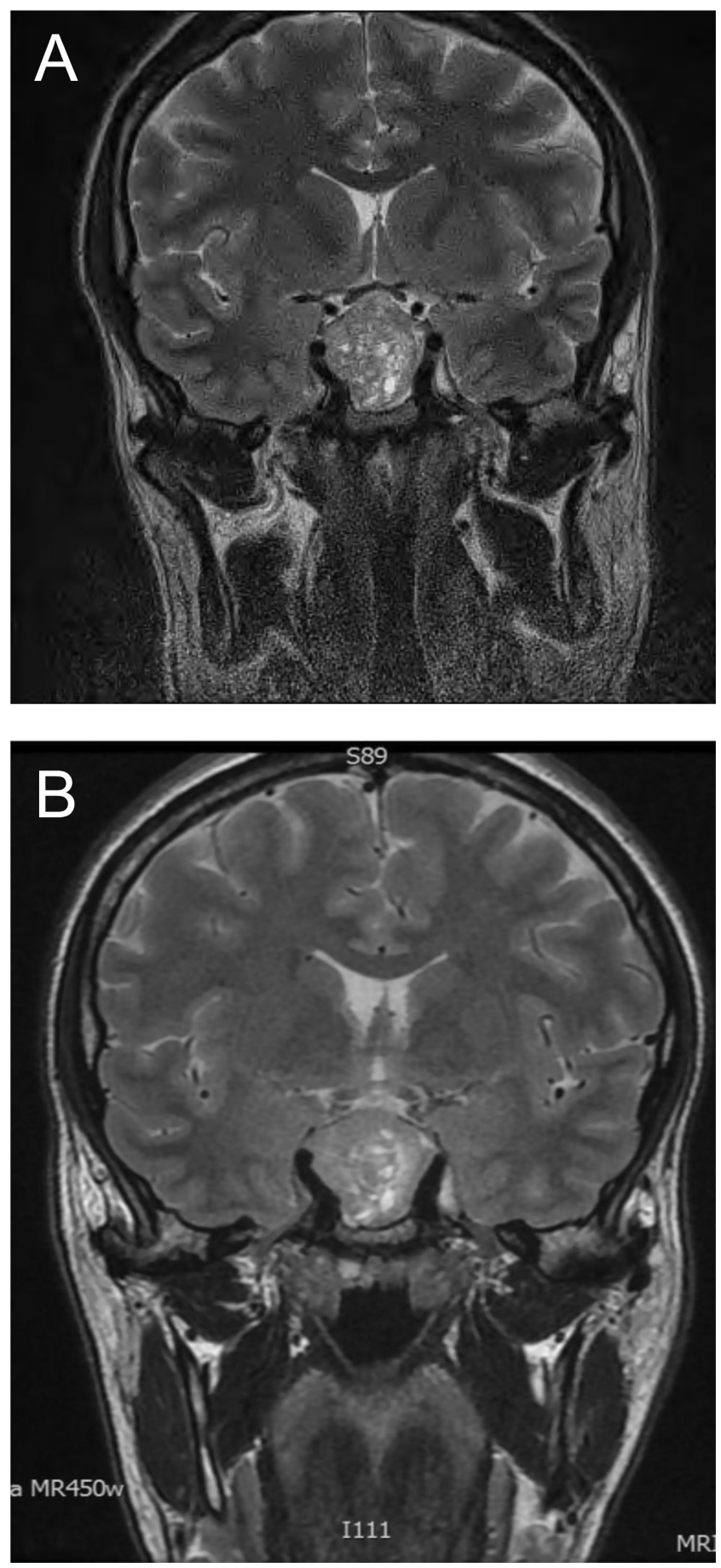

Figure 1

(A) T2 pituitary MRI at 12/40 - macroadenoma extending upwards into the suprasellar region causing distortion of the optic chiasm at 12 weeks gestation. (B) T2 pituitary MRI at $20 / 403.2 \times 2.7 \times 2.8 \mathrm{~cm}$ macroadenoma extending upwards into the suprasellar region causing compression of the optic chiasm at 20 weeks' gestation.

of the superior anti-tumour effects of octreotide, she was initiated on $100 \mu \mathrm{g}$ octreotide subcutaneously tds. Her visual field deficit completely recovered on repeat visual 
Table 1 Baseline endocrine laboratory evaluations.

\begin{tabular}{|c|c|c|c|}
\hline & Results at $12 / 40$ & Post-partum & Normal range \\
\hline T4 (pmol/L) & 17.6 & 17.1 & $12-22$ \\
\hline $\mathrm{TSH}(\mathrm{mIU} / \mathrm{L})$ & 0.7 & 0.63 & $0.4-3.8$ \\
\hline IGF-1 (nmol/L) & 82 & 60 & $9-33$ \\
\hline Prolactin (mU/L) & 827 & 557 & $102-496$ \\
\hline $\mathrm{GH}(\mu \mathrm{g} / \mathrm{L})$ & 65 & 9.81 & \\
\hline $\begin{array}{l}\text { Random cortisol } \\
(\mathrm{nmol} / \mathrm{L})\end{array}$ & 116 & 99 & \\
\hline
\end{tabular}

field testing after 2 weeks. At 24/40 gestation there was a further deterioration in her visual fields and her octreotide dose was increased to $150 \mu \mathrm{g}$ tds. She once again had a resolution of her visual field defect to normal within 2 weeks. Her vision remained stable for the remainder of pregnancy on the increased dose.

\section{Outcome and follow-up}

She was diagnosed with gestational diabetes when screened at $14 / 40$. She was initially treated with dietary modifications, but was commenced on basal bolus insulin regimen at 22/40 due to elevated fasting capillary glucose readings. She remained normotensive throughout pregnancy.

20 week's Gestation LE

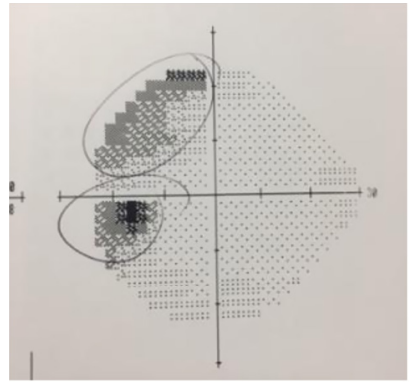

24 weeks Gestation LE

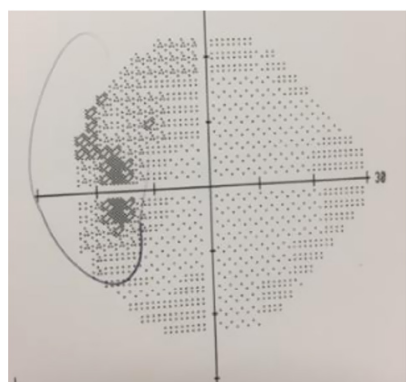

20 week's Gestation RE

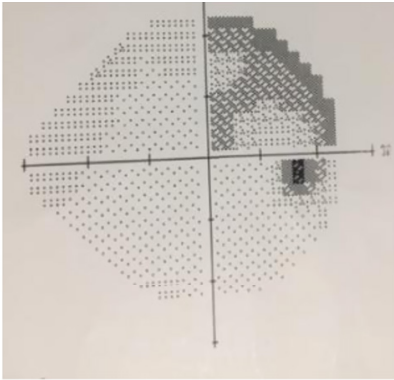

24 weeks Gestation RE

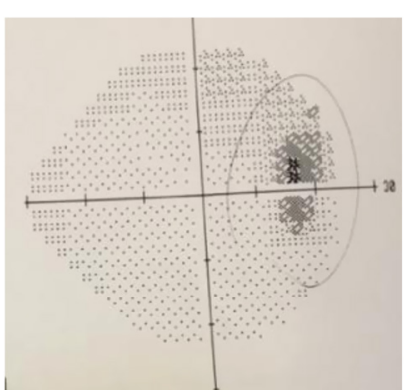

Foetal growth continued along the 50th centile throughout pregnancy. She underwent an elective caesarean section at 34/40, foetal weight was $3.2 \mathrm{~kg}$ at birth with an APGAR score of 9. The neonate was examined by an experienced neonatologist and no congenital abnormalities were identified. She opted not to breastfeed and she is menstruating regularly post-partum. Her biochemical assessment post-partum showed a mildly elevated serum prolactin concentration of $557 \mathrm{mU} / \mathrm{L}$ (102-496) and normal thyroid function (Table 1). Her random GH and plasma IGF-1 concentrations were significantly elevated at $9.81 \mu \mathrm{g} / \mathrm{L}$ and $60 \mathrm{nmol} / \mathrm{L}$ (normal range: 9-33) respectively.

Post-partum, she discontinued all medication for diabetes mellitus, her repeat OGTT showed impaired glucose tolerance and her HbA1c was controlled at $44 \mathrm{~mol} /$ mol with no medication. Her ACTH axis was normal when it was evaluated in the post-partum period, she had a peak cortisol of $527 \mathrm{nmol} / \mathrm{L}$ on an insulin tolerance test. She was commenced on octreotide LAR $40 \mathrm{mg}$ and referred for surgery. Immunohistochemical analysis of her adenoma showed a sparsely granulated tumour which stained positively for $\mathrm{GH}$ and sparse staining of prolactin. The adenoma did not stain for FSH, LH, TSH or ACTH.

\section{Post 100micg octreotide LE-22/40}

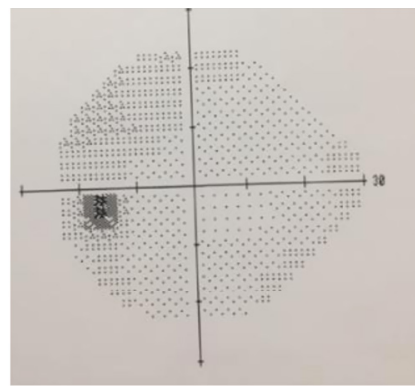

Post 150micg octreotide LE-26/40

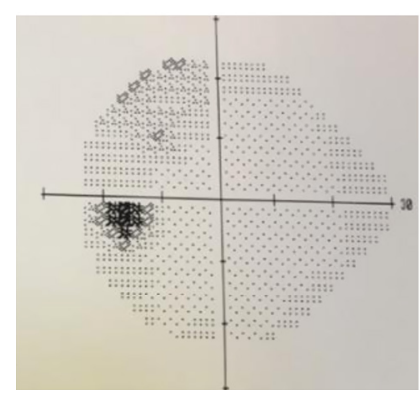

Post 100micg octreotide $\mathrm{RE}-22 / 40$

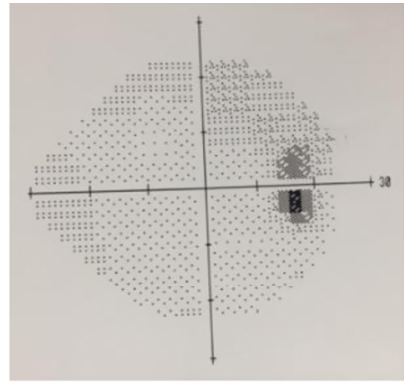

Post 150micg octreotide RE-26/40

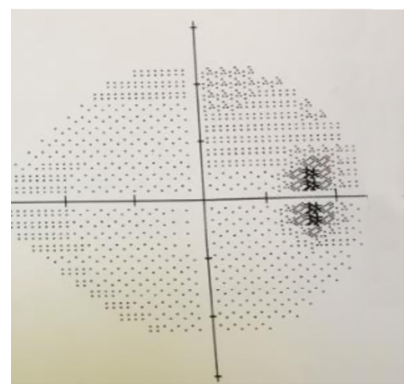

Figure 2

Formal perimetry testing throughout pregnancy - demonstrating a dramatic improvement in bitemporal hemianopia following the introduction of octreotide. LE, left eye; RE, right eye. 
She had persistent hyper-secretion post-operatively and monthly octreotide LAR was restarted. She has not yet been screened for an AIP mutation. At last follow-up, the infant has been developing normally at age 2 years.

\section{Discussion}

Acromegaly is a low prevalence disease characterised by growth hormone hyper-secretion. Subfertility is common in acromegaly and has numerous aetiologies, both as a result of the pituitary adenoma but also the treatments involved to treat the disease resulting in hypogonadism. Therefore, pregnancy in acromegaly is rare with less than 200 cases reported in the literature. The limited data that are available suggest that pregnancy in acromegaly is generally safe $(1,3,4)$; however, there have been case reports of tumour expansion and the development of visual field deficits during pregnancy (5).

The largest series of pregnancy in acromegaly is derived from the French acromegaly registry, which published pregnancy outcomes in 59 pregnancies in 46 women. Four of 59 pregnancies in this cohort were complicated by clinically apparent tumour expansion; 3 of these were women who were diagnosed with acromegaly whilst pregnant. None of these four women were treated with a somatostatin analogue, one received glucocorticoids, two received glucocorticoid therapy and bromocriptine and one had surgical intervention in the second trimester (1). However, other case series have not demonstrated any visual field deficits in spite of the withdrawal of medical therapy (6). As tumour expansion during pregnancy is rare, the approach to the pregnant acromegaly patient with tumour expansion is guided only by available case reports.

The goals of management of acromegaly shift during pregnancy. Both maternal and foetal perspectives need to be considered. The Endocrine Society Guidelines recommend withdrawing medical treatment before pregnancy and monitoring visual fields in each trimester (7). In exceptional cases where tumour expansion occurs, the options for treatment include both medical and surgical approaches. In this case, our patient declined surgery and was therefore offered medical therapy as an alternative to preserve her visual fields.

During normal pregnancy, placental GH, which bears significant homology with $22 \mathrm{kDa}$ pituitary $\mathrm{GH}$, is released in increasing amounts throughout the pregnancy. Some assays may cross-react with placental GH, therefore, when interpreting GH results during pregnancy, one needs to consider the specificity of the assay used (8). Furthermore, routine measurement of $\mathrm{GH}$ concentrations during pregnancy is not usually indicated as it will not change the management of the patient.

Medical therapy for tumour shrinkage in acromegaly consists of dopamine agonists and somatostatin analogues (9). However, neither cabergoline nor somatostatin analogues are licenced in pregnancy, and the risk/benefit profile should be carefully examined before deciding on a therapy. The little data available regarding the safety of somatostatin analogue therapy during pregnancy are from isolated case reports and small case series. The main concern with the use of somatostatin analogues in pregnancy is the risk of foetal growth restriction which was noted in case reports of women continuing medication during pregnancy (1). Somatostatin analogues have been shown to cross the placenta by passive diffusion (10) and there is evidence that short-acting octreotide produces a reversible reduction in uterine artery blood flow on Doppler flow analysis, which may explain the case reports of growth restriction in foetuses exposed to somatostatin analogues (11).

Trans-sphenoidal surgery during pregnancy is rare. The French series had one second trimester surgery in 59 pregnancies (1). Jallad et al. reported outcomes in 27 pregnancies with acromegaly, 3 patients in this cohort underwent surgery in the second trimester due to visual field loss. One of these three pregnancies miscarried shortly after surgery, the other two delivered healthy babies at term (3). Other case reports have reported surgery in the second trimester due to increased intracranial pressure (12), apoplexy (13) and visual loss (1, 14, 15). Surgery during pregnancy is typically performed in the second trimester. However, there are inherent surgical and anaesthetic risks that need to be considered before undergoing the procedure and surgery in the second trimester is associated with increased rate of foetal loss (16). It is crucial therefore that these patients are managed by a multi-disciplinary team including an endocrinologist, neurosurgeon, neuro-radiologist and ophthalmologist to ensure optimal outcomes for both the mother and the foetus.

Whilst there are reports of patients who had rescue of visual fields with dopamine agonist therapy during pregnancy (17), this is the first case report of resolution of visual field compromise with somatostatin analogues that we, the authors are aware of. This case report would suggest that somatostatin analogue therapy may be an alternative option to surgery for patients with symptomatic tumour growth during pregnancy. 


\section{Declaration of interest}

The authors declare that there is no conflict of interest that could be perceived as prejudicing the impartiality of the research reported.

\section{Funding}

This research did not receive any specific grant from any funding agency in the public, commercial or not-for-profit sector.

\section{Patient consent}

Written informed consent has been obtained from the patient for publication of the submitted article and accompanying images.

\section{Author contribution statement}

A M Hannon wrote the first draft. I F, G K and D O H provided direct care for the patient. All authors contributed to editing of the manuscript.

\section{References}

1 Caron P, Broussaud S, Bertherat J, Borson-Chazot F, Brue T, CortetRudelli C \& Chanson P. Acromegaly and pregnancy: a retrospective multicenter study of 59 pregnancies in 46 women. Journal of Clinical Endocrinology and Metabolism 201095 4680-4687. (https://doi. org/10.1210/jc.2009-2331)

2 Cheng V, Faiman C, Kennedy L, Khoury F, Hatipoglu B, Weil R \& Hamrahian A. Pregnancy and acromegaly: a review. Pituitary 201215 59-63. (https://doi.org/10.1007/s11102-011-0330-3)

3 Jallad RS, Shimon I, Fraenkel M, Medvedovsky V, Akirov A, Duarte FH \& Bronstein MD. Outcome of pregnancies in a large cohort of women with acromegaly. Clinical Endocrinology $2018 \mathbf{8 8}$ 896-907. (https://doi.org/10.1111/cen.13599)

4 Hannon AM, O'Shea T, Thompson CA, Hannon MJ, Dineen R, Khattak AK, Gibney J, O'Halloran DJ, Hunter S, Thompson CJ, et al. Pregnancy in acromegaly is safe and is associated with improvements in GH/IGF-1 concentrations. European Journal of Endocrinology 2019 180 K21-K29. (https://doi.org/10.1530/EJE-18-0688)

5 Kasuki L, Neto LV, Takiya CM \& Gadelha MR. Growth of an aggressive tumor during pregnancy in an acromegalic patient. Endocrine Journal 201259 313-319. (https://doi.org/10.1507/endocrj. EJ11-0306)

6 Dias M, Boguszewski C, Gadelha M, Kasuki L, Musolino N, Vieira JG \& Abucham J. Acromegaly and pregnancy: a prospective study. European Journal of Endocrinology 2014170 301-310. (https://doi. org/10.1530/EJE-13-0460)
7 Katznelson L, Laws ER, Jr, Melmed S, Molitch ME, Murad MH, Utz A, Wass JA \& Endocrine Society. Acromegaly: an endocrine society clinical practice guideline. Journal of Clinical Endocrinology and Metabolism 201499 3933-3951. (https://doi.org/10.1210/jc.20142700)

8 Junnila RK, Strasburger CJ \& Bidlingmaier M. Pitfalls of insulinlike growth factor-I and growth hormone assays. Endocrinology and Metabolism Clinics of North America 201544 27-34. (https://doi. org/10.1016/j.ecl.2014.10.003)

9 Bevan JS, Atkin SL, Atkinson AB, Bouloux PM, Hanna F, Harris PE, James RA, McConnell M, Roberts GA, Scanlon MF, et al. Primary medical therapy for acromegaly: an open, prospective, multicenter study of the effects of subcutaneous and intramuscular slow-release octreotide on growth hormone, insulin-like growth factor-I, and tumor size. Journal of Clinical Endocrinology and Metabolism $2002 \mathbf{8 7}$ 4554-4563. (https://doi.org/10.1210/jc.2001-012012)

10 Caron P, Gerbeau C \& Pradayrol L. Maternal-fetal transfer of octreotide. New England Journal of Medicine 1995333 601-602. (https://doi.org/10.1056/NEJM199508313330918)

11 Maffei P, Tamagno G, Nardelli GB, Videau C, Menegazzo C, Milan G, Calcagno A, Martini C, Vettor R, Epelbaum J, et al. Effects of octreotide exposure during pregnancy in acromegaly. Clinical Endocrinology 201072 668-677. (https://doi.org/10.1111/j.13652265.2009.03706.x)

12 Montini M, Pagani G, Gianola D, Pagani MD, Piolini R \& Camboni MG. Acromegaly and primary amenorrhea: ovulation and pregnancy induced by SMS 201-995 and bromocriptine. Journal of Endocrinological Investigation 199013 193. (https://doi.org/10.1007/ BF03349537)

13 Atmaca A, Dagdelen S \& Erbas T. Follow-up of pregnancy in acromegalic women: different presentations and outcomes. Experimental and Clinical Endocrinology and Diabetes 2006114 135-139. (https://doi.org/10.1055/s-2005-873004)

14 Guven S, Durukan T, Berker M, Basaran A, Saygan-Karamursel B $\&$ Palaoglu S. A case of acromegaly in pregnancy: concomitant transsphenoidal adenomectomy and cesarean section. Journal of Maternal-Fetal and Neonatal Medicine 200619 69-71. (https://doi. org/10.1080/14767050500434021)

15 Mozas J, Ocon E, Lopez de la Torre M, Suarez AM, Miranda JA \& Herruzo AJ. Successful pregnancy in a woman with acromegaly treated with somatostatin analog (octreotide) prior to surgical resection. International Journal of Gynecology and Obstetrics 199965 71-73. (https://doi.org/10.1016/S0020-7292(98)00221-5)

16 Cohen-Kerem R, Railton C, Oren D, Lishner M \& Koren G. Pregnancy outcome following non-obstetric surgical intervention. American Journal of Surgery 2005190 467-473. (https://doi. org/10.1016/j.amjsurg.2005.03.033)

17 Yap AS, Clouston WM, Mortimer RH \& Drake RF. Acromegaly first diagnosed in pregnancy: the role of bromocriptine therapy. American Journal of Obstetrics and Gynecology 1990163 477-478. (https://doi. org/10.1016/0002-9378(90)91178-F)

Received in final form 29 March 2019

Accepted 8 April 2019 